\title{
Classificação geotécnica de solos e sustentabilidade: estudo aplicado à região metropolitana de Belo Horizonte
}

Os sistemas de classificação geotécnica de solos são ferramentas imprescindiveis para proposição de soluções de pavimentação racionais e sustentáveis. Por prover os critérios de seleção dos materiais, essas metodologias impactam diretamente na viabilidade técnica, ambiental e econômica dos projetos. O presente artigo apresenta uma análise estatística comparativa entre os sistemas de classificação de solos mais utilizados no Brasil, contrastando os conceitos de classificação da escola rodoviária americana e os procedimentos desenvolvidos nacionalmente, pautados na consideração das particularidades do ambiente tropical. Foram coletadas onze amostras de diferentes unidades pedológicas da Região Metropolitana de Belo Horizonte (RMBH) e realizaram-se ensaios de caracterização TRB/AASHTO e segundo a metodologia MCT/G-MCT. As amostras foram submetidas aos ensaios de caracterização mecânica - CBR/Expansão, MiniCBR/RIS, Triaxiais de Cargas Repetidas - cujos resultados foram utilizados como parâmetro de comparação com o comportamento previsto pelos métodos classificatórios. Em linhas gerais, constataram-se resultados mais assertivos para os ensaios da metodologia MCT/G-MCT. Foi observado grau de associação significativo entre os parâmetros classificatórios dessa metodologi - sobretudo d' e índice RIS - e o desempenho mecânico-geotécnico medido. Por outro lado, identificaram-se mecanismos de classificação falhos em relação à metodologia TRB aos solos estudados. Os dados obtidos de Índice de Grupo (IG) e Índice de Plasticidade (IP), importantes parâmetros de classificação dessa metodologia, e a resposta mecânica dos solos avaliados apresentaram baixa inter-relação. Além de se estabelecer comparação semi-quantitativa entre parâmetros classificatórios e mecânicos, o grau de associação entre os mesmos foi quantificado por procedimento estatístico, através do cálculo de coeficiente de correlação de Pearson. Dessa forma, apresenta-se elementos para discussão da adequabilidade e compatibilidade dos sistemas de classificação de solos para fins rodoviários para regiões tropicais.

Palavras-chave: Sustentabilidade; Classificação de solos; Pavimentação; MCT; Solos tropicais.

\section{Geotechnical classification of soils and sustainability: a study applied to the metropolitan region of Belo Horizonte}

\begin{abstract}
Soil geotechnical classification systems are essential tools for proposing rational and sustainable paving solutions. By providing material selection criteria, these methodologies directly impact Soil geotechnical classification systems are essential tools for proposing rational and sustainable paving solutions. By providing material selection criteria, these methodologies directly impact contrasting the concepts of American system and the procedures developed regionally, based on the particularities of the tropical environment. Eleven samples from different pedological contrasting the concepts of American system and the procedures developed regionally, based on the particularities of the tropical environment. Eleven samples from different pedological
units of the Metropolitan Region of Belo Horizonte (RMBH) were collected, TRB/AASHTO and MCT characterization tests were performed. The samples were submitted to mechanical tests CBR/Expansion, Mini-CBR/RIS, Repeated Load Triaxial - whose results were used as a parameter of comparison with the behavior predicted by the classificatory methods. In general, terms, more assertive results were found for the MCT methodology. It was observed a significant degree of association between the classificatory parameters of this methodology - mainly d' and RIS index - and the measured mechanical-geotechnical performance. On the other hand, we identified faulty classification mechanisms in relation to the TRB methodology to the soils studied. The data obtained from the Group Index (GI) and Plasticity Index (PI), important parameters of classification of this methodology, and the mechanical response of the evaluated soils presented statistical procedure, using the Pearson correlation coefficient. Thus, we present elements to discuss the suitability and compatibility of soil classification systems for road use in tropical regions.
\end{abstract}

Keywords: Sustainability; Soil classification; Paving; MCT; Tropical Soils.

Topic: Sustentabilidade nos Transportes

Reviewed anonymously in the process of blind peer.
Received: 02/08/2020

Approved: 19/09/2020
Bruno Oliveira da Silva (iD)

Universidade Federal de Ouro Preto, Brasil http://lattes.cnpq.br/8292888983921544

http://orcid.org/0000-0003-0014-7304

bruolisilva@yahoo.com.br

Eleonardo Lucas Pereira (ic

Universidade Federal de Ouro Preto, Brasil

http://lattes.cnpq.br/6474173159047103

http://orcid.org/0000-0001-9772-3050

eleonardo@ufop.edu.br

Geraldo Oliveira Marques (ic

Universidade Federal de Juiz de Fora, Brasil

http://lattes.cnpq.br/4503245454189660

http://orcid.org/0000-0002-2601-2646

geraldo.marques@ufjf.edu.br
Marlilene Silva Gomes

Universidade Federal de Juiz de Fora, Brasil

http://lattes.cnpq.br/9158129648792302

http://orcid.org/0000-0001-5141-8288

marlilene.gomes@engenharia.ufif.br
Referencing this:

SILVA, B. O.; PEREIRA, E. L.; MARQUES, G. L. O.; GOMES, M. S..

Classificação geotécnica de solos e sustentabilidade: estudo aplicado à região metropolitana de Belo Horizonte. Revista Ibero Americana de Ciências Ambientais, v.11, n.5, p.362-375, 2020. DOI: http://doi.org/10.6008/CBPC2179-6858.2020.005.0034 


\section{INTRODUÇÃO}

Obras de estradas se caracterizam pelas grandes extensões longitudinais e transposição de diferentes unidades geomorfológicas, onde ocorrem extensa variedade de solos e agregados para construção. Os estudos de caracterização do subleito e de seleção de jazidas, contemplando toda essa diversidade, constituem etapa essencial para a proposição de projetos de pavimentação que sejam viáveis em termos ambientais, técnicos e econômicos. Nesse contexto, os procedimentos de classificação geotécnica de solos se apresentam como uma ferramenta de grande utilidade: a identificação de índices físicos permite o agrupamento da extensa variedade de materiais em classes que apresentam características semelhantes em relação ao comportamento mecânico-geotécnico (SANTOS, 2006).

A expansão da malha viária do estado de Minas Gerais ocorrida a partir de 2004, no contexto do programa ProAcesso, possibilitou a constatação do esgotamento das jazidas naturais de agregados, inclusive nas regiões de baixa concentração da malha viária pavimentada (MEDRADO, 2009). Segundo esse estudo, os recursos foram amplamente empregados, durante muitos anos, em serviços de conservação de rodovias com revestimento primário. $\mathrm{O}$ autor conclui que a utilização extensiva desse tipo de material inviabiliza as soluções de projeto, sobretudo para pavimentação de vias de baixo volume de tráfego, tornando imperativa a tarefa de utilização de materiais alternativos.

Os ensaios classificatórios e de avaliação do comportamento mecânico constituem uma importante ferramenta na seleção de materiais para construção de pavimentos. O uso de ferramentas estatísticas simples, pode ser muito útil na aferição da qualidade dos sistemas classificatórios no que se refere à qualidade de predição do comportamento mecânico dos solos. O cálculo de parâmetros estatísticos, envolvendo dados de caracterização e comportamento mecânico, confere caráter objetivo para avaliação da assertividade das metodologias de classificação.

Avaliar a adequabilidade das metodologias que norteiam a seleção de materiais para construção de pavimentos é vital para contribuir com seu caráter de sustentabilidade. Nesse contexto, este estudo objetiva avaliar por meio do coeficiente de correlação de Pearson, a adequabilidade dos sistemas TRB/AASHTO, MCT/G-MCT para classificação de onze amostras de solos coletados na Região Metropolitana de Belo Horizonte (RMBH). A análise será feita em termos do grau de inter-relação entre os parâmetros classificatórios desses sistemas e índices mecânicos obtidos dos ensaios CBR e triaxial de carga repetida. Salienta-se o fato de que a RMBH está inserida em uma localidade intensamente explorada do ponto de vista de seus recursos naturais, além da carência de trabalhos que abordam a caracterização geotécnica rodoviária dos solos desta região.

\section{REVISÃO TEÓRICA}

No cenário da pavimentação nacional, é comum o uso de técnicas tradicionais para a seleção de materiais para projetos de pavimentos, como o sistema de classificação Transportation Research Board (TRB/AASHTO), importado de entidades norte-americanas. Desenvolvido em regiões com a presença de solos 
constituídos em clima frio e temperado, o sistema se baseia nas propriedades físicas do solo (granulometria, limite de liquidez (LL), índice de plasticidade (IP) e índice de grupo (IG)) como parâmetros de análise para a sua classificação.

Entretanto, a metodologia apresenta algumas incompatibilidades nos estudos dos solos tropicais, como discutido em Silva et al. (2010) e Achterberg (2014). Tais técnicas frequentemente apontam para a seleção de agregados naturais ou britados e desprezam as potencialidades de solos lateríticos finos, abundantes em boa parte do território nacional.

Diante das dificuldades de aplicação das premissas classificatórias americanas aos solos do ambiente tropical, surgiu a metodologia MCT (Miniatura, Compactada, Tropical) para a classificação de solos. Desenvolvida por Nogami et al. (1981), foi resultado de anos de experimentação e observação do comportamento in situ de obras no estado de São Paulo. A metodologia envolve ensaios para a determinação de propriedades que refletem o comportamento mecânico e hidráulico dos solos tropicais e a sua classificação em lateríticos e não lateríticos. A metodologia MCT utiliza os ensaios de Compactação Mini-MCV e Perda de Massa por Imersão para determinar o índice de argilosidade dos solos ( $c^{\prime}$ ) e o índice de laterização dos solos (e'), e em seguida cruzá-los no ábaco classificatório, para definição da classe do solo em análise.

Motivados pela experiência bem-sucedida no interior paulista, vários autores buscaram estender a aplicação da MCT a outras regiões do Brasil. Castro (2002) e Marangon (2004) relatam excelentes resultados com a utilização da metodologia para classificação de solos de diferentes regiões de Minas Gerais. Silva et al. (2011) estudou solos de Viçosa-MG e constatou que aqueles que possuíam comportamento laterítico, apresentavam características geotécnicas compatíveis para o seu emprego em pavimentos de rodovias de baixo volume de tráfego. De acordo com Achterberg (2014), solos de Santa Cruz do Sul-RS descartados pela metodologia americana para uso em obras de rodovias, podem ser utilizados segundo a metodologia MCT. Roza (2018), encontrou bons resultados para o comportamento mecânico em amostras de solos na região norte do estado do Mato Grosso.

De forma a complementar a classificação MCT, Villibor et al. (2017) propuseram uma metodologia que objetiva a caracterização dos solos tropicais de granulação grossa, denominada G- MCT, G de granular e MCT em função do procedimento já consagrado de caracterização da matriz de finos. Em linhas gerais, esse procedimento consiste em cruzar as informações obtidas dos ensaios de classificação da MCT com a análise granulométrica, especificamente com os percentuais passantes nas peneiras $\mathrm{N}^{\circ} 10$ e $\mathrm{N}^{\circ} 200$. Dessa forma, supera-se parcialmente a limitação imposta pela metodologia original, que trata apenas de partículas menores que $2,00 \mathrm{~mm}$.

As metodologias de classificação de solos apresentadas constituem uma ferramenta de grande utilidade, uma vez que possibilita o agrupamento da extensa variedade de materiais em classes que apresentam características semelhantes em relação ao comportamento mecânico-geotécnico (SANTOS, 2006). Se tratando dos quesitos para o dimensionamento de pavimentos, o ensaio CBR (California Bearing Ratio) e ensaio triaxial de cargas repetidas para obtenção do módulo de resiliência (MR), são utilizados para a determinação do comportamento mecânico do solo, sendo o primeiro um parâmetro empírico e o segundo 
de caráter mecanístico.

No ensaio CBR, obtém-se a capacidade de suporte de solos e agregados por meio da aplicação de um carregamento de forma lenta e crescente, provocando um estado de ruptura plástica caracterizada por grandes deslocamentos. Entretanto, a forma de aplicação do carregamento neste ensaio pouco se assemelha ao que ocorre na realidade com a estrutura do pavimento, visto que, a estrutura é submetida a um regime de cargas repetidas, transientes, com diferentes intensidades e frequências e que, na maioria das vezes, provocam pequenos deslocamentos (BERNUCCl et al., 2008).

De acordo com Medina et al. (2015), ensaios de carga repetida, cuja intensidade do carregamento varia ao longo do tempo e sua aplicação ocorre em regime cíclico, constituem a melhor configuração para reprodução parcial da complexidade de condições que existem em campo. Nesse sentido, um importante parâmetro mecânico é o módulo de resiliência (MR), que pode ser obtido por meio de ensaios laboratoriais e procedimentos de campo. O MR é uma grandeza que retrata a intensidade das deformações recuperáveis de um material, face a intensidade do carregamento aplicado. Valores elevados de MR, denotam pouca deformabilidade do material sob determinado estado de tensão induzido pelo carregamento, enquanto valores baixos indicam material com muita deformabilidade, característica indesejável à pavimentação.

\section{MATERIAIS E MÉTODOS}

O desenvolvimento desta pesquisa ocorreu em quatro etapas, quais sejam: (i) pesquisa de mapas pedológicos da RMBH; (ii) com base nestes mapas, definição dos pontos de coleta e amostragem; (iii) realização dos ensaios de caracterização segundo a metodologia TRB/AASHTO, MCT/G-MCT; (iv) realização dos ensaios de caracterização mecânica, segundo as metodologias CBR e de ensaios triaxiais de carga repetida. De posse dos dados obtidos na campanha experimental avaliou-se, por meio do coeficiente de correlação de Pearson, qual metodologia classificatória tem maior assertividade na predição do comportamento mecânico medido em laboratório.

\section{Plano de amostragem}

Visando identificar as principais ocorrências pedológicas na região de estudo, consultou-se o mapa desenvolvido pela Universidade Federal de Minas Gerais e disponibilizado na plataforma digital do Plano Metropolitano da RMBH. Esse material foi útil para o conhecimento da distribuição espacial das unidades pedológicas na região de estudo e para definição preliminar do número de amostras e das possíveis áreas de coletas.

A Erro! Fonte de referência não encontrada.Erro! Fonte de referência não encontrada. mostra o mapa pedológico da Região Metropolitana estudado com os pontos de amostragem. Constata-se que RMBH é composta por solos lateríticos e de acordo com o viés pedológico os solos dessa região podem ser subdivididos em latossolos, solos podzólicos, cambissolos e litossolo, sendo que, os três primeiros constituem a quase totalidade da área de estudo. As maiores áreas são ocupadas pelos solos podzólicos vermelho escuros e podzólicos vermelho amarelos, variedades de argissolo. 
Observa-se também que, com exceção da ocorrência na região norte da RMBH, os litossolos estão dispostos de forma a acompanhar os perímetros das ocorrências do grupo latossolo ferrífero. De acordo com as informações coletadas neste mapa, definiu-se o quantitativo dos pontos de coleta de modo a contemplar os principais grupos pedológicos, definindo assim 11 pontos de amostragem.

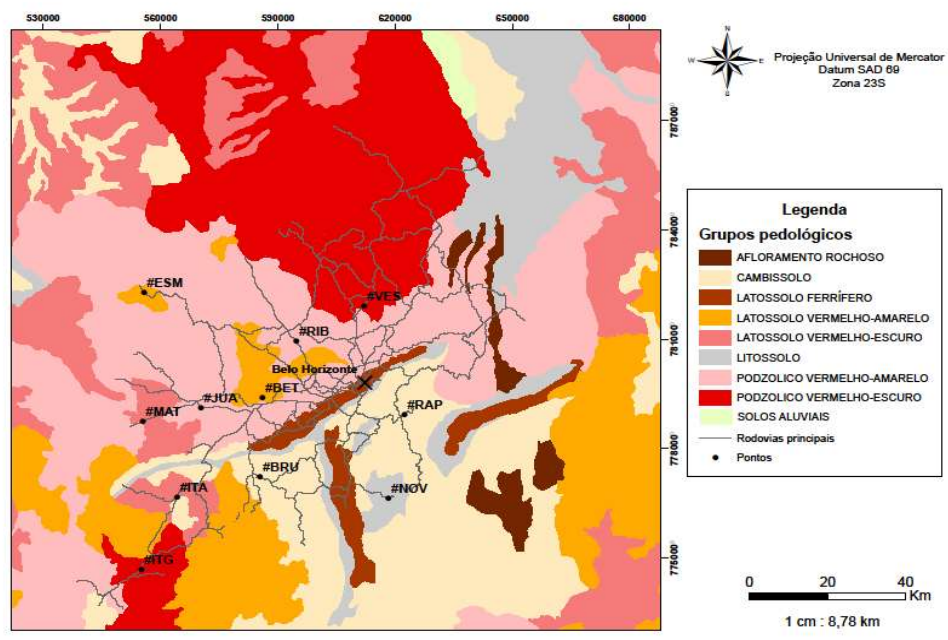

Figura 1: Mapa pedológico da RMBH e locação dos pontos de amostragem.

A Tabela 1 apresenta as principais informações dos pontos de amostragem. A identificação das amostras foi feita com notação referenciada ao município de onde foi coletada, como pode-se observar nas colunas 'ID' e 'Municípios' da presente tabela. Por exemplo: a amostra coletada no município de Vespasiano recebeu o 'ID' \#VES.

Tabela 1: Dados principais sobre os pontos de coleta.

\begin{tabular}{|c|c|c|c|c|c|}
\hline \multirow{2}{*}{ ID } & \multirow{2}{*}{ GRUPO PEDOLÓGICO PRESUMIDO } & \multirow{2}{*}{ RODOVIA } & \multirow{2}{*}{ MUNICÍPIO } & \multicolumn{2}{|c|}{ COORDENADAS } \\
\hline & & & & LATITUDE & LONGITUDE \\
\hline \#VES & Podzólico Vermelho Escuro & MG-010 & Vespasiano & $19^{\circ} 43^{\prime} 13.50^{\prime \prime S}$ & $43^{\circ} 55^{\prime} 51.95^{\prime \prime} \mathrm{O}$ \\
\hline \#RAP & Cambissolo & AMG-150 & Raposos & $19^{\circ} 59^{\prime} 16.62^{\prime \prime} \mathrm{S}$ & $43^{\circ} 49^{\prime} 47.52^{\prime \prime O}$ \\
\hline \#ITA & Latossolo Vermelho Escuro & MG-431 & Itatiauçu & $20^{\circ} 11^{\prime} 42.66^{\prime \prime} \mathrm{S}$ & $44^{\circ} 23^{\prime} 07.44^{\prime \prime} \mathrm{O}$ \\
\hline \#ITG & Podzólico Vermelho Escuro & BR-381 & Itaguara & $20^{\circ} 22^{\prime} 29.71 " \mathrm{~S}$ & $44^{\circ} 28^{\prime} 22.34^{\prime \prime O}$ \\
\hline \#MAT & Latossolo Vermelho Escuro & MG-050 & Mateus Leme & $20^{\circ} 00^{\prime} 49.63^{\prime \prime S}$ & $44^{\circ} 28^{\prime} 58.29^{\prime \prime O}$ \\
\hline \#JUA & Podzólico Vermelho Amarelo & BR 262 & Juatuba & $19^{\circ} 58^{\prime} 30.34^{\prime \prime} \mathrm{S}$ & $44^{\circ} 19^{\prime} 40.85^{\prime \prime O}$ \\
\hline \#ESM & Latossolo Vermelho Amarelo & MG-060 & Esmeraldas & $19^{\circ} 41^{\prime} 23.70^{\prime \prime} \mathrm{S}$ & $44^{\circ} 28^{\prime} 04.22^{\prime \prime O}$ \\
\hline \#BRU & Cambissolo & Estrada Vicinal & Brumadinho & $20^{\circ} 08^{\prime} 36.90^{\prime \prime S}$ & $44^{\circ} 10^{\prime} 58.53^{\prime \prime O}$ \\
\hline \#RIB & Podzólico Vermelho Amarelo & BR-040 & Rib.das Neves & $19^{\circ} 48^{\prime} 27.24^{\prime \prime S}$ & $44^{\circ} 05^{\prime} 45.34^{\prime \prime O}$ \\
\hline \#NOV & Litossolo & $B R-356$ & Nova Lima & $20^{\circ} 11^{\prime} 39.41 " \mathrm{~S}$ & $43^{\circ} 52^{\prime} 03.34^{\prime \prime} \mathrm{O}$ \\
\hline \#BET & Latossolo Vermelho Amarelo & Contorno/381 & Betim & $19^{\circ} 57^{\prime} 58.28^{\prime \prime} \mathrm{S}$ & $44^{\circ} 09^{\prime} 34.55^{\prime \prime} \mathrm{O}$ \\
\hline
\end{tabular}

\section{Ensaios de caracterização}

As amostras coletadas foram devidamente homogeneizadas e preparadas conforme prescrito na norma referência (NBR 6457/2016). A caracterização física foi realizada segundo dois procedimentos distintos. O primeiro procedimento foi por meio dos ensaios do sistema TRB/AASHTO: determinação da massa específica real dos grãos (NBR 6458/2016), limites de consistência (NBR 6459/2016 e NBR 7180/2016) e análise granulométrica (NBR 7181/2016).

Enquanto o segundo foi por meio dos ensaios da metodologia classificatória MCT: compactação MiniMCV (DNER-ME 256/1994) e ensaio de Perda de Massa por Imersão (DNER-ME 258/1994). Também foram 
considerados, os procedimentos da metodologia G-MCT (VILLIBOR e ALVES, 2017), que consistem em uma avaliação conjunta entre os resultados dos ensaios tradicionais da MCT e análise granulométrica.

\section{Ensaios mecânicos}

Após caracterização segundo as metodologias MCT/G-MCT e TRB/AASHTO, as amostras foram submetidas aos ensaios de determinação do índice CBR e expansão, segundo a normatização proposta pela DNIT 172/2016 - ME, Mini-CBR (DNER-ME 254/1997) e o módulo de resiliência, conforme a norma DNIT 134/2017 - ME.

Adotou-se energia de compactação intermediária para moldagem dos corpos de prova. Para a determinação do MR, foi realizado o ensaio triaxial de carga repetida, com corpos de prova de $10 \mathrm{~cm}$ de diâmetro por $20 \mathrm{~cm}$ de altura. Neste ensaio, foi aplicado um carregamento com frequência de $1 \mathrm{~Hz}$, sendo 0,1 segundo o tempo de aplicação da carga seguida de um tempo de descarregamento de 0,9 segundos. Os resultados foram analisados segundo as metodologias consagradas no meio técnico nacional: tensão confinante, tensão desvio e modelo composto.

Os ensaios Mini-CBR foram realizados nas condições imersa e não imersa, com o intuito de se obter a relação entre índices de suporte (RIS) conforme definido por Nogami et al. (1981). Esse parâmetro é obtido pela razão entre o valor de Mini-CBR imerso e não imerso e constitui grande recurso para diferenciação de solos de comportamento laterítico e não laterítico. Em cada amostra, realizou-se o ensaio em duas condições: (i) na umidade de moldagem, logo após a etapa de compactação Mini-Proctor; e (ii) após período de imersão com duração de 24 horas. Solos lateríticos apresentam pouca diferença entre os valores obtidos nas condições imersa e não imersa, consequentemente altos valores de RIS, enquanto solos não lateríticos apresentam queda acentuada de suporte na condição pós imersão.

\section{Análise estatística}

De posse dos resultados dos ensaios de caracterização e dos parâmetros mecânicos, utilizou-se de recurso estatístico para quantificação do grau de correspondência entre a resposta mecânica e os parâmetros das metodologias de classificação utilizadas. Por meio do cálculo do coeficiente de correlação de Pearson Equação 1 - pode-se quantificar o nível de associação entre parâmetros classificatórios e de comportamento mecânico.

$$
\rho=\frac{\sum_{i-1}^{n}\left(x_{i}-\bar{x}\right)\left(y_{i}-\bar{y}\right)}{\sqrt{\sum_{i=1}^{n}\left(x_{i}-\bar{x}\right)^{2}} \sqrt{\sum_{i=1}^{n}\left(y_{i}-\bar{y}\right)^{2}}}
$$

Em que: $x_{i}$ : valor da variável independente (parâmetros classificatórios);

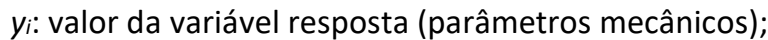
$\bar{x}$ : média das variáveis independentes; $\bar{y}$ : média das variáveis resposta.

Foram utilizados como parâmetros classificatórios para análise estatística, variáveis obtidas na metodologia MCT (índice de argilosidade dos solos $\left(c^{\prime}\right)$, inclinação do ramo seco da curva de compactação Mini-MCV - 12 golpes ( $\left.d^{\prime}\right)$, Perda de massa por imersão (Pi) e o índice RIS) e variáveis obtidas na metodologia TRB/AASHTO (Índice de Plasticidade (IP), Índice de Grupo (IG) e percentual passante nas peneiras №10, №40 
e $\left.\mathrm{N}^{\circ} 200\right)$.

As variáveis resposta consideradas, que se referem aos parâmetros mecânicos, foram o índice CBR (\%), expansão (\%) e os valores médio de MR medidos nos ensaios triaxiais de carga repetida. Calculou-se o coeficiente de correlação de Pearson para cada um dos pares possíveis de parâmetro classificatório e variável resposta mecânica.

\section{RESULTADOS}

\section{Ensaios de classificação TRB/AASHTO}

A Tabela 2 apresenta a síntese dos resultados dos ensaios de caracterização da metodologia TRB/AASHTO e a Erro! Fonte de referência não encontrada. apresenta o resultado do ensaio de granulometria, ambos para as onze amostras estudadas.

Tabela 2: Resumo dos resultados dos ensaios de caracterização TRB/AASHTO.

\begin{tabular}{|c|c|c|c|c|c|c|c|c|c|c|c|c|}
\hline \multirow{2}{*}{ Amostra } & \multirow{2}{*}{$\begin{array}{c}\gamma_{s} \\
\left(k N / m^{3}\right)\end{array}$} & \multicolumn{6}{|c|}{ Percentual passante } & \multirow{2}{*}{ LL (\%) } & \multirow{2}{*}{ IP (\%) } & \multirow{2}{*}{ IG } & \multirow{2}{*}{$\begin{array}{l}\text { Grupo } \\
\text { AASHTO }\end{array}$} & \multirow[t]{2}{*}{ TRB/ } \\
\hline & & $\# 10$ & $(2,00 \mathrm{~mm})$ & $\# 40$ & $(0,42 \mathrm{~mm})$ & $\# 200$ & $(0,075 \mathrm{~mm})$ & & & & & \\
\hline \#BET & 26,87 & 98,80 & & 85,09 & & 38,43 & & $\mathrm{NL}$ & NP & 1 & $A-4$ & \\
\hline \#BRU & 26,73 & 87,90 & & 78,28 & & 47,42 & & 49 & 15 & 5 & A-7-5 & \\
\hline \#ESM & 26,52 & 98,00 & & 91,40 & & 68,30 & & 59 & 31 & 18 & $A-7-6$ & \\
\hline \#ITA & 27,25 & 95,90 & & 93,12 & & 77,25 & & 43 & 18 & 12 & A-7-6 & \\
\hline \#ITG & 26,43 & 99,50 & & 97,05 & & 86,67 & & 51 & 23 & 16 & A-7-6 & \\
\hline \#JUA & 27,05 & 79,20 & & 76,80 & & 68,83 & & 48 & 13 & 10 & $A-7-5$ & \\
\hline \#MAT & 26,32 & 99,10 & & 92,47 & & 68,43 & & 51 & 21 & 13 & $A-7-5$ & \\
\hline \#NOV & 32,90 & 47,20 & & 32,68 & & 15,80 & & $\mathrm{NL}$ & NP & 0 & $A-1-b$ & \\
\hline \#RAP & 28,06 & 54,50 & & 47,08 & & 38,24 & & NL & NP & 1 & $A-4$ & \\
\hline \#RIB & 26,62 & 99,80 & & 84,96 & & 39,62 & & NL & NP & 1 & $A-4$ & \\
\hline \#VES & 26,90 & 60,00 & & 46,24 & & 33,91 & & 43 & 17 & 2 & $A-2-7$ & \\
\hline
\end{tabular}

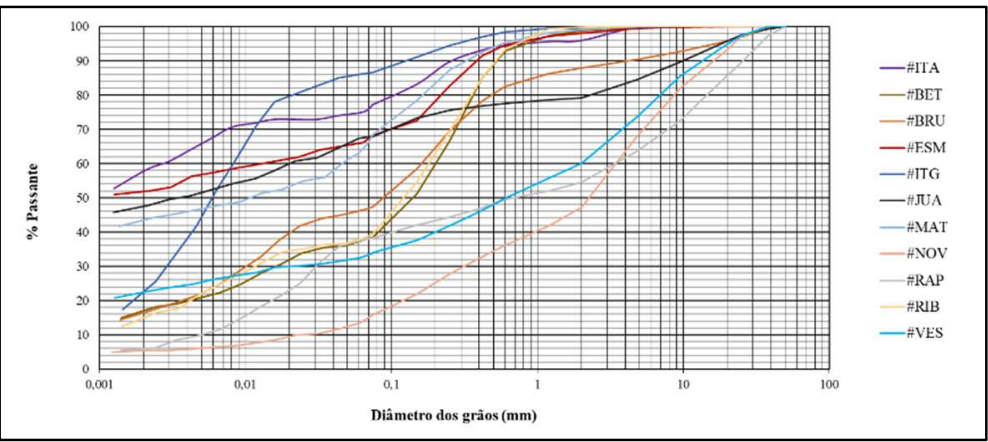

Figura 2: Curvas granulométricas das amostras em estudo.

\section{Ensaios de classificação MCT/G-MCT}

A

Tabela 3 mostra os resultados sintetizados das classificações MCT/G-MCT.

Tabela 3: Síntese dos resultados dos ensaios de classificação MCT/G-MCT.

\begin{tabular}{|c|c|c|c|c|c|c|c|c|c|c|c|}
\hline \multirow{2}{*}{ Amostra } & \multicolumn{2}{|c|}{ Mini-MCV } & \multirow{2}{*}{$\mathrm{Pi}(\%)$} & \multirow{2}{*}{$e^{\prime}$} & \multirow{2}{*}{ Grupo MCT } & \multirow{2}{*}{ \%pass \#10 } & \multirow{2}{*}{$\%$ pass \#200 } & \multirow{2}{*}{ Grupo } & \multirow{2}{*}{ G-MCT } & \multirow{2}{*}{ Grupo } & \multirow{2}{*}{ TRB/AASHTO } \\
\hline & $c^{\prime}$ & d' $^{\prime}$ & & & & & & & & & \\
\hline \#BET & 1,21 & 20,5 & 116 & 1,29 & $\mathrm{NA}^{\prime}$ & 98,8 & 38,43 & Gf-NA' & & A-4 & \\
\hline \#BRU & 1,26 & 13,4 & 120 & 1,39 & NS' & 87,9 & 47,42 & Gf-NS' & & $A-7-5$ & \\
\hline \#ESM & 2,26 & 47,7 & 30 & 0,90 & $L^{\prime} G^{\prime}$ & 98,0 & 68,30 & Gf-LG' & & $A-7-6$ & \\
\hline \#ITA & 1,82 & 24,2 & 60 & 1,13 & LG' & 95,9 & 77,25 & Gf-LG' & & $A-7-6$ & \\
\hline \#ITG & 1,43 & 6,52 & 325 & 1,85 & NS' & 99,5 & 86,67 & Gf-NS' & & $A-7-6$ & \\
\hline
\end{tabular}




\begin{tabular}{llllllllll} 
\#JUA & 1,94 & 27,5 & 60 & 1,10 & LG'$^{\prime}$ & 79,2 & 68,83 & Gf-LG' & A-7-5 \\
\#MAT & 1,66 & 43,8 & 40 & 0,95 & LG'$^{\prime}$ & 99,1 & 68,43 & Gf-LG' & A-7-5 \\
\#NOV & 1,29 & 58,0 & 85 & 1,06 & LA' $^{\prime}$ & 47,2 & 15,80 & Ps-LA' & A-1-b \\
\#RAP & 0,90 & 5,67 & 262 & 1,83 & NS' $^{\prime}$ & 54,5 & 38,24 & Gf-NS' & A-4 \\
\#RIB & 1,15 & 8,48 & 160 & 1,58 & NS' $^{\prime}$ & 99,8 & 39,62 & Gf-NS' & A-4 \\
\#VES & 2,01 & 42,1 & 90 & 1,11 & LG' $^{\prime}$ & 60,0 & 33,91 & Gf-LG' & A-2-7 \\
\hline
\end{tabular}

\section{Ensaios CBR/Expansão}

A Tabela 4 apresenta os resultados dos ensaios CBR e expansão das onze amostras estudadas, bem como os parâmetros da compactação. Em conjunto com os outros resultados da caracterização mecânica, os valores de CBR/expansão serão avaliados à luz dos sistemas de classificação utilizados, no sentido de se avaliar a qualidade desses em predizer sobre o comportamento mecânico medido.

Tabela 4: Síntese dos resultados dos ensaios de CBR e expansão.

\begin{tabular}{lllll}
\hline \multirow{2}{*}{ Amostra } & \multicolumn{1}{c}{ Parâmetrqs da compactação } & CBR & (\%) & Expansão \\
\cline { 2 - 5 } & \multicolumn{2}{l}{ (\%) } \\
\hline \#BET & 14,4 & 17,89 & 16,6 & 0,65 \\
\#BRU & 19,5 & 15,64 & 20,7 & 1,31 \\
\#ESM & 22,2 & 16,14 & 15,5 & 0,01 \\
\#ITA & 25,2 & 15,54 & 11,7 & 0,01 \\
\#ITG & 15,8 & 16,00 & 7,3 & 2,33 \\
\#JUA & 24,5 & 15,33 & 29,1 & 0,05 \\
\#MAT & 23,0 & 15,55 & 23,4 & 0,09 \\
\#NOV & 11,5 & 21,78 & 51,3 & 0,01 \\
\#RAP & 18,5 & 16,93 & 19,8 & 1,08 \\
\#RIB & 19,1 & 16,50 & 13,7 & 1,43 \\
\#VES & 14,6 & 18,50 & 29,0 & 0,49 \\
\hline
\end{tabular}

\section{Ensaios Mini-CBR e o índice RIS}

Os ensaios Mini-CBR foram realizados com o objetivo principal de avaliar a magnitude da perda de suporte dos solos estudados após período de imersão, por meio do índice RIS. Em cada amostra, realizou-se o ensaio em duas condições, quais sejam: (i) na umidade de moldagem, logo após a etapa de compactação Mini-Proctor (Mini-CBR Ho) e (ii) após período de imersão com duração de 24 horas (Mini-CBR Is). A realização desse procedimento pode ser muito útil no sentido de complementar os ensaios classificatórios da MCT, uma vez que confere um importante parâmetro de diferenciação entre solos de comportamento laterítico e não laterítico. A

Tabela 5 mostra os resultados dos ensaios Mini-CBR, bem como as respectivas relações RIS obtidas.

Tabela 5: Parâmetros de compactação e valores de índice Mini-CBR e índice RIS.

\begin{tabular}{|c|c|c|c|c|}
\hline \multirow{2}{*}{ Amostra } & Parâmetros da compactação & \multirow{2}{*}{ Mini - CBR но (\%) } & \multirow{2}{*}{ Mini - CBR Is (\%) } & \multirow{2}{*}{ RIS (\%) } \\
\hline & wy $(\alpha / \mathrm{d})$ máx $\left(\mathrm{kN} / \mathrm{m}^{3}\right)$ & & & \\
\hline \#BET & $14,1 \quad 18,05$ & 47,0 & 13,8 & 29 \\
\hline \#BRU & $19,5 \quad 15,84$ & 29,0 & 5,5 & 19 \\
\hline \#ESM & 16,46 & 36,0 & 19,0 & 53 \\
\hline \#ITA & 16,45 & 26,0 & 16,2 & 62 \\
\hline \#ITG & 15,00 & 27,0 & 1,2 & 4 \\
\hline \#JUA & 16,15 & 44,9 & 25,3 & 56 \\
\hline \#MAT & 16,54 & 40,5 & 21,0 & 52 \\
\hline \#NOV & 22,43 & 62,0 & 49,0 & 79 \\
\hline \#RAP & 16,81 & 27,5 & 1,9 & 7 \\
\hline \#RIB & 17,05 & 29,9 & 9,8 & 33 \\
\hline \#VES & 18,18 & 38,0 & 21,0 & 55 \\
\hline
\end{tabular}




\section{Módulo de resiliência}

A Tabela 6 apresenta os resultados dos ensaios triaxiais de cargas repetidas - parâmetros $k$ e respectivos valores de $R^{2}$ dos modelos de comportamento do $M R$ em função da tensão confinante $\left(O_{3}\right)$, tensão desvio $\left(\sigma_{d}\right)$ e modelo composto - das onze amostras ensaiadas. Para obtenção do modelo composto, utilizou-se uma planilha eletrônica similar à desenvolvida por Ferreira (2002). Salienta-se que os corpos de prova foram moldados na condição de umidade ótima da energia de compactação intermediária.

Tabela 6: Resultados da modelagem do MR.

\begin{tabular}{|c|c|c|c|c|c|c|c|c|c|c|}
\hline \multirow{2}{*}{ Amostra } & \multicolumn{3}{|c|}{$M R=k_{1} \sigma_{d}^{k_{2}}$} & \multicolumn{3}{|c|}{$M R=k_{1} \sigma_{3}^{k_{2}}$} & \multicolumn{4}{|c|}{$M R=k_{1} \sigma_{3}^{k_{2}} \sigma_{d}^{k_{3}}$} \\
\hline & & & $\mathbf{R}^{2}$ & & & $\mathbf{R}^{2}$ & & & & $\mathbf{R}^{2}$ \\
\hline \#BET & 109,4 & $-0,2644$ & 0,63 & $124,7^{1}$ & $-0,1640$ & 0,16 & 166,2 & 0,3145 & $-0,4799$ & 0,84 \\
\hline \#BRU & 47,2 & $-0,6020$ & 0,91 & 39,3 & $-0,5414$ & 0,49 & 61,2 & 0,1959 & $-0,7354$ & 0,91 \\
\hline \#ESM & 101,4 & $-0,4001$ & 0,87 & 91,9 & $-0,3512$ & 0,45 & 124,6 & 0,1541 & $-0,5039$ & 0,80 \\
\hline \#ITA & 24,9 & $-0,5532$ & 0,44 & 24,2 & $-0,4479$ & 0,19 & 23,8 & 0,3663 & $-0,9952$ & 0,88 \\
\hline \#ITG & 48,6 & $-0,5677$ & 0,94 & 39,0 & $-0,5267$ & 0,54 & 57,8 & 0,1328 & $-0,6596$ & 0,88 \\
\hline \#JUA & 123,9 & $-0,3755$ & 0,90 & 117,9 & $-0,3148$ & 0,42 & 159,1 & 0,1892 & $-0,5044$ & 0,90 \\
\hline \#MAT & 129,5 & $-0,4167$ & 0,92 & 113,3 & $-0,3768$ & 0,50 & 153,8 & 0,1297 & $-0,5049$ & 0,88 \\
\hline \#NOV & 555,0 & 0,0457 & 0,02 & 945,2 & 0,2214 & 0,27 & 1155,0 & 0,5236 & $-0,2915$ & 0,55 \\
\hline \#RAP & 54,1 & $-0,3757$ & 0,75 & 58,5 & $-0,2704$ & 0,26 & 84,2 & 0,3301 & $-0,5981$ & 0,88 \\
\hline \#RIB & 24,2 & $-0,6488$ & 0,93 & 19,7 & $-0,5859$ & 0,51 & 31,8 & 0,2017 & $-0,785$ & 0,91 \\
\hline \#VES & 294,6 & $-0,3083$ & 0,77 & 301,1 & $-0,2314$ & 0,33 & 346,2 & 0,1204 & $-0,390$ & 0,80 \\
\hline
\end{tabular}

\section{Análise estatística}

O procedimento estatístico aplicado neste estudo consiste em calcular o coeficiente de correlação de Pearson para todos os pares possíveis de índices classificatórios e mecânicos. Os parâmetros de caracterização são os parâmetros c', d', Pi (\%), RIS (\%) - da metodologia MCT - e IP (\%), IG e percentual passante nas peneiras $N^{\circ} 10, N^{\circ} 40$ e $N^{\circ} 200$ - da metodologia TRB/AASHTO. Já os parâmetros de resposta considerados são os valores médios do MR, índice CBR e expansão.

A

Tabela 7 mostra os valores dos coeficientes de correlação de Pearson calculados para todos os pares, compostos por um parâmetro classificatório e outro do comportamento mecânico. O coeficiente varia entre -1 (correlação negativa perfeita) e 1 (correlação positiva perfeita). A tabela de cores indica o grau de associação entre os parâmetros, conforme Santos (2007).

Tabela 7: Coeficientes de correlação de Pearson para os pares entre coeficientes classificatórios e mecânicos

\begin{tabular}{|c|c|c|c|}
\hline Coeficientes de correlação de Pearson & MR médio & CBR & Expansão \\
\hline$c^{\prime}$ & 0,376723 & 0,030772 & $-0,452361$ \\
\hline $\mathbf{d}^{\prime}$ & 0,720278 & 0,687212 & $-0,785417$ \\
\hline $\mathbf{P i}$ & $-0,386307$ & $-0,383348$ & 0,753440 \\
\hline RIS & 0,582977 & 0,639989 & $-0,777542$ \\
\hline IP & 0,070260 & $-0,343170$ & 0,199295 \\
\hline IG & $-0,168796$ & $-0,428709$ & 0,283170 \\
\hline Pass10 & $-0,541230$ & $-0,736182$ & 0,365546 \\
\hline Pass40 & $-0,563932$ & $-0,753490$ & 0,368810 \\
\hline Pass200 & $-0,389746$ & $-0,631681$ & 0,342650 \\
\hline \multicolumn{4}{|l|}{ Legenda (SANTOS, 2007) } \\
\hline $0<|\rho|<0,1$ & \multicolumn{3}{|c|}{ Correlação ruim } \\
\hline $0,1 \leq|\rho|<0,5$ & \multicolumn{3}{|c|}{ Correlação fraca } \\
\hline
\end{tabular}


$0,5 \leq|\rho|<0,8$

$0,8 \leq|\rho|<1,0$

\section{DISCUSSÃO}

\section{Ensaios de classificação TRB/AASHTO}

A massa específica real dos grãos representa uma característica intrínseca das partículas que compõe o solo analisado e é função do peso específico molecular de seus componentes. De acordo com Pinto (2006), solos ricos em quartzos (areia), apresentam peso específico de $26,5 \mathrm{kN} / \mathrm{m}^{3}$, enquanto argilas lateríticas podem apresentar valores da ordem de $30 \mathrm{kN} / \mathrm{m}^{3}$, devido à presença de minerais ricos em elementos de alto peso molecular, como o ferro. Observa-se na Tabela 2 que a maioria das amostras de solos utilizadas, apresentam valores de peso específico acima de $26,5 \mathrm{kN} / \mathrm{m}^{3}$ e no caso da amostra \#NOV acima de $30 \mathrm{kN} / \mathrm{m}^{3}$, o que indica a presença de elementos de alto peso molecular na composição deste solo, uma característica dos solos lateríticos.

De acordo com o que foi observado na Tabela 2 é possível constatar a predominância de solos finos, uma vez que, apenas as amostras \#NOV e \#VES apresentam percentual passante na peneira \#200 (0,075 mm) menor que 35\%, se caracterizando como um solo granular, de acordo com o critério de classificação TRB/AASHTO. Verifica-se também a predominância de amostras pertencentes a classe A-7, sendo seis ocorrências nesse grupo. Em seguida, três amostras do grupo A-4, uma do grupo A-2 e uma do grupo A-1. Entretanto, avaliando o procedimento de análise utilizado pelo sistema classificatório TRB/AASHTO e os resultados obtidos para as amostras estudadas, constata-se duas inconsistências importantes.

A primeira diz respeito ao fato das amostras \#BRU e \#ITG serem enquadradas no grupo A-7, junto a outros quatro solos de granulometria marcadamente argilosa. A amostra \#BRU é constituída majoritariamente pela fração areia (42\%) e a \#ITG pela fração silte (63\%). Os resultados obtidos indicam que a metodologia apresenta mecanismo classificatório falho, uma vez que inclui solos de granulometrias distintas em um mesmo grupo. Salienta-se que a granulometria é um dos parâmetros de diferenciação mais importantes adotados pelo sistema.

A segunda inconsistência refere-se à amostra \#RAP: esse solo foi classificado como A-4, em conjunto com as amostras \#RIB e \#BET. Analisando os resultados dos limites de consistência e o percentual que passa na peneira $\mathrm{N}^{\circ} 200$ das três amostras, de fato observa-se muita semelhança. No entanto, ao compararmos as outras frações granulométricas de \#RAP em relação à \#RIB e \#BET, constata-se diferença significativa. A primeira amostra é constituída majoritariamente por pedregulhos e silte (as duas frações juntas somam 78\%) enquanto as outras duas amostras são predominantemente arenosas. Nesse ponto, o mecanismo de falha no procedimento classificatório descrito no parágrafo anterior se repete.

A análise dos resultados obtidos permite constatar que se atribui peso classificatório excessivo ao percentual passante na peneira $\mathrm{N}^{\circ} 200$, desprezando a contribuição das outras frações na constituição da estrutura geral dos solos. Observa-se que, para as amostras estudadas, os limites definidos para os percentuais passantes parecem inadequados. Por exemplo, a exigência de material passante na peneira 
$\mathrm{N}^{\circ} 200$ ser maior que $36 \%$ para os grupos A-7, incluem em uma mesma classe os solos \#BRU e \#ITG que apresentam, respectivamente, $47 \%$ e $87 \%$ passantes nessa peneira.

\section{Ensaios de classificação MCT/G-MCT}

Uma primeira constatação notável é que não há correspondência direta entre estes procedimentos classificatórios e a metodologia TRB/AASHTO. Mais que isso, pode-se afirmar que as metodologias apresentam alto grau de incompatibilidade entre si, uma vez que amostras classificadas em um mesmo grupo TRB/AASHTO são classificadas em grupos antagônicos da MCT/G-MCT. Por exemplo, as amostras \#ITA/\#ESM e \#ITG pertencem ao grupo A-7-6 e, pela metodologia G-MCT, aos grupos Gf-LG' e Gf-NS', respectivamente. Ou seja, a metodologia proposta para solos tropicais indica que as amostras em questão têm comportamento geotécnico completamente distinto - um laterítico e outro não laterítico - ao contrário do que prediz a metodologia americana. A mesma inconsistência relatada ocorre com os pares (\#BRU - \#MAT) e (\#RAP/\#RIB - \#BET).

A incompatibilidade relatada entre os sistemas era esperada, uma vez que essas metodologias foram desenvolvidas em conformidade a condições de meio físico completamente diversas. A diferenciação dos solos conforme sua gênese não constitui um critério classificatório da metodologia americana. Em função disso, solos de comportamento laterítico e não laterítico podem ser situados em um mesmo grupo classificatório neste sistema. Essa observação desvenda uma consequência grave advinda da utilização deste procedimento classificatório à realidade brasileira: solos tropicais, submetidos à classificação TRB/AASHTO, pode apresentar comportamento geotécnico melhor ou pior que aquele esperado por esse sistema. Incompatibilidades como esta também foram relatadas por como discutido em Silva et al. (2010) e Achterberg (2014), em seus estudos. Em outras palavras, pode-se desprezar um material bom e, em contrapartida, selecionar um material que apresente propriedades aquém das esperadas.

\section{Ensaios CBR/Expansão}

Segundo os valores de referência do Manual de Pavimentação (DNIT, 2006), com exceção de \#ITG, todas amostras atendem aos requisitos técnicos para composição de subleitos estradais, apresentando CBR $\geq 2 \%$ e expansão menor ou igual a $2 \%$. Pelo critério da expansão, as amostras \#BRU, \#ITG, \#RAP e \#RIB não são adequadas para construção de camadas de reforço do subleito, sub-base e base, pois esse parâmetro foi maior que $1,0 \%$.

Nenhum material estudado reúne as características técnicas requeridas pelo DNIT para a construção de camadas de base ( $C B R \geq 80 \%$, expansão menor ou igual a 0,5\%, $L L \leq 25 \%$ e IP $\leq 6$ ). Teoricamente, o único material que poderia ser aproveitado como sub-base é o solo coletado em Nova Lima (\#NOV), uma vez que apresenta $C B R \geq 20 \%$, expansão menor ou igual a $1 \%$ e IG=0.

\section{Ensaios Mini-CBR e o índice RIS}

A avaliação da magnitude da perda de suporte dos solos estudados após período de imersão, por 
meio do índice RIS, pode ser muito útil no sentido de complementar os ensaios classificatórios da MCT, uma vez que confere um importante parâmetro de diferenciação entre solos de comportamento laterítico e não laterítico.

Os valores de Mini-CBR foram calculados através das equações que constam na norma referência DNER-ME 254/1997 - desenvolvidas por Nogami (1972, citado por MARSON, 2004). Essas relações foram obtidas através de procedimento de ajuste estatístico, com o tratamento dos dados de um grande número de ensaios e desenvolvimento de correlação direta entre os índices CBR e Mini-CBR. Conforme será apresentado posteriormente, na análise estatística, os resultados dos índices RIS serão tratados mais como parâmetro de diferenciação entre solos lateríticos e não lateríticos do que como um índice mecânico.

\section{Módulo de resiliência}

O módulo de resiliência de solos arenosos apresenta maior dependência em relação aos níveis de tensão de confinamento $\left(\sigma_{3}\right)$, ao passo que os argilosos da tensão desvio $\left(\sigma_{d}\right)$ (MEDINA et al., 2015). Os resultados obtidos nessa pesquisa sugerem que não há correspondência direta entre a qualidade de ajuste do modelo (aqui representada pelo valor do parâmetro $\mathrm{R}^{2}$ ) e a predominância granulométrica das amostras. Os resultados das amostras predominantemente arenosas (\#BET, \#BRU e \#RIB) apresentaram melhor ajuste ao modelo $\sigma_{d}$, que teoricamente é mais apropriado a solos argilosos.

Esses resultados podem ser justificados pela presença, ainda que em menor porcentagem, de fração argila na composição dos solos ensaiados. Em nove das onze amostras, obteve-se um percentual igual ou superior a $15 \%$ dessa fração, o que supostamente influenciou no comportamento resiliente observado. A única amostra com $\mathrm{R}^{2}$ favorável ao modelo $\sigma_{3}$ foi a \#NOV, de caráter mais arenoso. No entanto, o valor do coeficiente de determinação obtido foi muito baixo, indicando má qualidade do modelo ajustado.

Com exceção da amostra \#NOV, todos as outras amostras apresentaram $R^{2} \geq 0,80$ na análise do modelo composto. De acordo com Medina et al. (2015), o modelo composto, em geral, tende a apresentar valores maiores de $\mathrm{R}^{2}$, ao considerar o efeito combinado das tensões confinantes e desviadoras, porém isto não significa que este modelo seja o mais adequado para todos os tipos de solo. Por exemplo, as amostras \#ESM, \#ITG, \#MAT e \#RIB, obteve-se valores de $\mathrm{R}^{2}$ superiores para o modelo em função da tensão desvio, em comparação com o modelo composto. Roza (2018), também relata a ocorrência de baixos valores de $R^{2}$ para o modelo composto em sua pesquisa, com solos lateríticos do Mato Grosso.

\section{Análise estatística}

A análise dos resultados obtidos permite algumas constatações. Os valores de d' e RIS apresentam correlação moderada com todos os parâmetros mecânico-geotécnicos (MR médio, CBR e expansão). A metodologia TRB/AASHTO confere grande peso ao IG para estimativa dos valores de suporte CBR, logo os valores deveriam apresentar grau de associação moderado ou alto, o que não foi observado para os solos ensaiados. Esse fato pode ser explicado pela diferença de gênese entre os solos de climas frios e temperados e os solos tropicais. Além do IG, outro parâmetro importante é o IP, e ambos não estão significativamente 
associados a nenhum parâmetro resposta avaliado. Tal constatação também sugere que essa metodologia apresenta baixo potencial de classificação para os solos estudados.

O comportamento expansivo dos solos é razoavelmente associado aos parâmetros d', Pi e o índice RIS. Sabe-se que a magnitude dos valores de expansão está fortemente relacionada à constituição mineralógica dos solos que, por sua vez, está diretamente ligada ao seu grau de laterização. Portanto, podese concluir que essa constatação corrobora as expectativas relacionadas à metodologia $\mathrm{MCT}$, uma vez que o principal critério de classificação é a diferenciação dos solos de comportamento laterítico (pouco expansivos) e não lateríticos (expansivos). Ainda, os parâmetros granulométricos e relacionados à plasticidade dos solos não estão associados à expansão, o que complementa e confirma a afirmação de que o comportamento expansivo está intimamente relacionado a questões referentes à gênese e constituição mineralógica dos solos, que são contempladas na metodologia MCT.

\section{CONCLUSÕES}

O presente estudo reuniu dados para discussão sobre a qualidade de metodologias de classificação de solos usuais no universo da pavimentação nacional. Atenção especial foi dedicada à comparação entre os sistemas TRB, metodologia americana amplamente utilizada no meio técnico brasileiro, e a MCT/G-MCT, sistema classificatório que considera as particularidades do meio físico tropical. A pesquisa foi fundamentada na avaliação dos resultados obtidos para solos da RMBH.

Pelo fato de não considerar as peculiaridades da pedogênese tropical, as ferramentas classificatórias da metodologia TRB mostraram-se incompatíveis para a avaliação da maior parte das amostras estudadas. Em relação à essa metodologia, destaca-se que o índice de grupo (IG) apresenta baixo grau de associação com as respostas mecânicas avaliadas. Chama a atenção o baixo grau de correlação entre esse parâmetro e os valores de suporte CBR, uma vez que ambos deveriam estar intrinsicamente relacionados, segundo a construção teórica do sistema TRB.

Os resultados obtidos sugerem que os parâmetros granulométricos considerados pela TRB (percentuais passantes nas peneiras \#10, \#40 e \#200) apresentam o maior nível de associação com a resposta mecânica dos materiais, sobretudo para o CBR e o módulo de resiliência médio (os valores de expansão não se associam significativamente a esses parâmetros). No entanto, a metodologia americana atribui peso classificatório exagerado ao percentual que passa na peneira $\mathrm{N}^{\circ} 200$, causando a inclusão de amostras com granulometrias muito distintas em uma mesma classe.

Em relação à metodologia $\mathrm{MCT}$, observou-se melhor relação entre os parâmetros classificatórios e o desempenho mecânico. Pelo menos para os solos estudados, a diferenciação do comportamento dos solos em laterítico ou não laterítico, se mostrou mais adequada que os critérios da TRB. Chama a atenção o bom grau de associação obtido entre os parâmetros mecânicos $C B R$, módulo de resiliência e expansão e os índices d' e RIS, o qual foi corroborado pela análise estatística.

As análises apresentadas indicam algumas inconsistências em relação à utilização do sistema TRB para classificação dos solos estudados. O procedimento classificatório da MCT, ao considerar as 
peculiaridades desses solos e do meio físico em que ocorrem, permite superar parte das dificuldades advindas da utilização daquela metodologia. As complementações da G-MCT, ao incorporar informações referentes à granulometria das amostras, potencializa o sistema de classificação de solos tropicais, fornecendo resultados coerentes e de grande utilidade para a classificação geotécnica de solos.

\section{REFERÊNCIAS}

ABNT. Associação Brasileira de Normas Técnicas. NBR 6457: Amostras de solo: Preparação para ensaios de compactação e ensaios de caracterização. São Paulo: ABNT, 2016.

ABNT. Associação Brasileira de Normas Técnicas. NBR 6458: Grãos de solos que passam na peneira de $4,8 \mathrm{~mm}$ : Determinação da massa específica. São Paulo: ABNT, 2016.

ABNT. Associação Brasileira de Normas Técnicas. NBR 6459: Solo: Determinação do limite de liquidez. São Paulo: ABNT, 2016.

ABNT. Associação Brasileira de Normas Técnicas. NBR 7180: Solo: Determinação do limite de plasticidade. São Paulo: ABNT, 2016.

ABNT. Associação Brasileira de Normas Técnicas. NBR 7181: Solo: Análise granulométrica. São Paulo: ABNT, 2016.

ACHTERBERG, H. A.. Estudo sobre a aplicação da classificação geotécnica MCT para um solo residual de Santa Cruz Do Sul/RS. Dissertação (Mestrado em Engenharia Civil) - Universidade de Santa Cruz do Sul, Santa Cruz do Sul, 2014.

BERNUCCI, L. B.; MOTTA, L. M.; CERATTI, J. A. P.; SOARES, J. B.. Pavimentação asfáltica: formação básica para engenheiros. Rio de Janeiro: Petrobrás, 2006.

CASTRO, B. M. C.. Caracterização geotécnica de solos da região central de Minas Gerais para aplicação em obras rodoviárias. Dissertação (Mestrado em Ciências em Engenharia Civil) - Universidade Federal do Rio de Janeiro, Rio de Janeiro, 2002.

DNER. Departamento Nacional de Estradas de Rodagem. ME 254: Solos compactados em equipamento miniatura: MiniCBR e expansão. Rio de Janeiro: DNER, 1997.

DNER. Departamento Nacional de Estradas de Rodagem. ME 256: Solos compactados em equipamento miniatura: determinação da perda de massa por imersão. Rio de Janeiro: DNER, 1994.

DNER. Departamento Nacional de Estradas de Rodagem. ME 258: Solos compactados em equipamento miniatura: MiniMCV. Rio de Janeiro: DNER, 1994.

DNIT. Departamento Nacional de Infraestrutura de Transportes. IPR 719: manual de pavimentação. Rio de Janeiro: DNIT, 2006.

DNIT. Departamento Nacional de Infraestrutura de Transportes. ME 172: Solos: Determinação do índice de suporte Califórnia utilizando amostras não trabalhadas. Rio de Janeiro: DNIT, 2016.

DNIT. Departamento Nacional de Infraestrutura de Transportes. ME 134: Pavimentação: Solos: Determinação do módulo de resiliência. Rio de Janeiro: DNIT, 2018.
Dissertação (Mestrado em Ciências em Engenharia Civil) Universidade Federal do Rio de Janeiro, Rio de Janeiro, 2002.

MARANGON, M.. Proposição de estruturas típicas de pavimentos para região de minas gerais utilizando solos lateríticos locais a partir da pedologia, classificação MCT e resiliência. Tese (Doutorado em Ciências em Engenharia Civil) - Universidade Federal do Rio de Janeiro, Rio de Janeiro, 2004.

MARSON, L. A.. Correlações entre ensaios CBR E Mini-CBR para solos lateríticos de textura fina. Dissertação (Mestrado em Infraestrutura de Transportes) - Instituto Tecnológico de Aeronáutica, São José dos Campos, 2004.

MEDINA, J.; MOTTA, L. M. G.. Mecânica dos Pavimentos. 3 ed. Rio de Janeiro: Interciência, 2015.

MEDRADO, W. A.. Caracterização geotécnica de solo da Região Norte de Minas Gerais para aplicação em obras Rodoviárias. Dissertação (Mestrado em Geotecnia) Universidade Federal de Ouro Preto, Ouro Preto, 2009.

NOGAMI, J. S.; VILLIBOR, D. F.. Uma Nova Classificação de Solos para Finalidades Rodoviárias. In: SIMPÓSIO BRASILEIRO DE SOLOS TROPICAIS EM ENGENHARIA. Anais. Rio de Janeiro: UFRJ, 1981.

PINTO, C. S.. Curso básico de mecânica dos solos. 3 ed. São Paulo: Oficina de Textos, 2006.

ROZA, A. E. D.. Contribuição para projeto mecanístico: empírico de pavimentos asfálticos na região norte do estado do Mato Grosso. Dissertação (Mestrado em Engenharia Civil) - Universidade Federal do Rio de Janeiro, Rio de Janeiro, 2018.

SANTOS, C.. Estatística descritiva: Manual de Autoaprendizagem. 2 ed. Lisboa: Sílabo, 2007.

SANTOS, E. F.. Estudo comparativo de diferentes sistemas de classificações geotécnicas aplicadas aos solos tropicais. Dissertação (Mestrado em Engenharia Civil) - Universidade de São Paulo, São Carlos, 2006.

SILVA, T. O.; CARVALHO, C. A. B.; LIMA, D. C.; CALIJURI, M. L.; LANI, J. L.; OLIVEIRA, T. M.. Sistemas de classificações geotécnicas de solos: estudo de caso aplicado à rodovia não pavimentada VCS 346, Viçosa, MG. Revista Árvore, Viçosa, v.34, n.2, p.313-321, 2010.

SILVA, T. O.; CARVALHO, C. A. B.; LIMA, D. C.; CALIJURI, M. L.; MACHADO, C. C.; OLIVEIRA, T. M.. Avaliação do subleito de rodovias vicinais de baixo volume de tráfego por meio de ensaios geotécnicos. Revista Árvore, Viçosa, v.35, n.4, p.825-833, 2011

VILLIBOR, D. F.; ALVES, D. M. L.. Classificação de solos tropicais de granulação fina e grossa. Revista Pavimentação, Rio de Janeiro, n.43, p.17-37, 2017. 
VILLIBOR, D. F.; NOGAMI, J. S.. Pavimentos econômicos:

tecnologia do uso dos solos finos lateríticos. São Paulo: Arte

\& Ciência, 2009.

A CBPC - Companhia Brasileira de Produção Científica (CNPJ: 11.221.422/0001-03) detém os direitos materiais desta publicação. Os direitos referem-se à publicação do trabalho em qualquer parte do mundo, incluindo os direitos às renovações, expansões e disseminações da contribuição, bem como outros direitos subsidiários. Todos os trabalhos publicados eletronicamente poderão posteriormente ser publicados em coletâneas impressas sob coordenação da Sustenere Publishing, da Companhia Brasileira de Produção Científica e seus parceiros autorizados. Os (as) autores (as) preservam os direitos autorais, mas não têm permissão para a publicação da contribuição em outro meio, impresso ou digital, em português ou em tradução. 